\title{
EPR experiments in LiTbF4, LiHoF4, and LiErF4 at submillimeter frequencies
}

\author{
Magariño, J.; Tuchendler, J.; Beauvillain, P.; Laursen, Ib
}

Published in:

Physical Review B

Link to article, DOI:

10.1103/PhysRevB.21.18

Publication date:

1980

Document Version

Publisher's PDF, also known as Version of record

Link back to DTU Orbit

Citation (APA):

Magariño, J., Tuchendler, J., Beauvillain, P., \& Laursen, I. (1980). EPR experiments in LiTbF4, LiHoF4, and LiErF4 at submillimeter frequencies. Physical Review B, 21(1), 18-28. https://doi.org/10.1103/PhysRevB.21.18

\section{General rights}

Copyright and moral rights for the publications made accessible in the public portal are retained by the authors and/or other copyright owners and it is a condition of accessing publications that users recognise and abide by the legal requirements associated with these rights.

- Users may download and print one copy of any publication from the public portal for the purpose of private study or research.

- You may not further distribute the material or use it for any profit-making activity or commercial gain

- You may freely distribute the URL identifying the publication in the public portal

If you believe that this document breaches copyright please contact us providing details, and we will remove access to the work immediately and investigate your claim. 


\title{
EPR experiments in $\mathrm{LiTbF}_{4}, \mathrm{LiHoF}_{4}$, and $\mathrm{LiErF}_{4}$ at submillimeter frequencies
}

\author{
J. Magariño and J. Tuchendler* \\ Laboratoire de Physique des Solides de l'Ecole Normale Superieure, ${ }^{\dagger} 24$ rue Lhomond, 75231 Paris, France \\ P. Beauvillain \\ Institut d'Electronique Fondamentale, ${ }^{\dagger}$ Batiment 220 , Universite Paris Sud, 91405 Orsay, France \\ I. Laursen \\ Department of Electrophysics, The Technical University of Denmark: DK 2800, Lyngby, Denmark
} (Received 12 March 1979)

\begin{abstract}
Electron-paramagnetic-resonance experiments in $\mathrm{LiTbF}_{4}, \mathrm{LiHoF}_{4}$, and $\mathrm{LiErF}_{4}$ have been performed at frequencies between 70 and $600 \mathrm{GHz}$, in magnetic fields up to $60 \mathrm{kG}$ and in the temperature range $1.4<T<25 \mathrm{~K}$. The experiments yield precise information on the lowest levels of the rare-earth ions. We investigated the resonance line position and the linewidth as a function of frequency and temperature. The $g$ values and the couplings between the lowest levels are determined. The local field around the magnetic ion is measured directly and the Curie constant $\Theta_{C}$ can be calculated. The results give the single-ion parameters appropriate to a description of the low-temperature magnetism in these compounds.
\end{abstract}

\section{INTRODUCTION}

The $\mathrm{LiY}_{1-x} R_{x} \mathrm{~F}_{4}$ series, where $R$ is a rare earth, have attracted considerable interest primarily because they are efficient laser host materials. ${ }^{1}$

In the dilute materials, $\operatorname{LiY}_{1-x} R_{x} \mathrm{~F}_{4}$, Sattler and Nemarich ${ }^{2}$ have observed the EPR spectra of $\mathrm{Nd}^{3+}$, $\mathrm{Dy}^{3+}, \mathrm{Er}^{3+}$, and $\mathrm{Yb}^{3+}$. The $\mathrm{Tb}^{3+}$ spectrum has been observed by Laursen and $\mathrm{Holmes}^{3}$ and the $\mathrm{Ho}^{3+}$ spectrum by Magariño et al. ${ }^{4}$

The magnetic properties of the $\mathrm{Li} R \mathrm{~F}_{4}$ compounds also present a great theoretical interest due to the simplicity of these materials. They consist of fairly ionic atoms with high-point tetragonal symmetry $\left(S_{4}\right)$, the rare earths being primarily coupled through dipolar forces.

The magnetic properties of $\mathrm{LiTbF}_{4}$ have first been observed by Holmes et al. ${ }^{5}$ by magnetic-susceptibility measurements. They showed that $\mathrm{LiTbF}_{4}$ is ferromagnetic below $T_{C}=2.86 \mathrm{~K}$ with the magnetic moment along the tetragonal $\vec{c}$ axis, and that the predominant contribution to the Curie-Weiss temperature, $\Theta_{C}$, comes from the dipolar forces. The precise comparison between dipolar and exchange interaction has been made by quasielastic-neutronscattering experiments. ${ }^{6}$ It confirmed that the dipolar interactions are more important than the exchange interactions.

$\mathrm{LiHoF}_{4}$ and $\mathrm{LiErF}_{4}$ have been studied by Hansen et al. ${ }^{7}$ Their magnetic-susceptibility measurements showed that $\mathrm{LiHoF}_{4}$ is ferromagnetic, with the magnetic moment along the $\overrightarrow{\mathrm{c}}$ axis: No ordering was observed in $\mathrm{LiErF}_{4}$ which was predicted by these au- thors to be ferromagnetic. The crystal-field parameters were determined in the two materials. The ferromagnetism in $\mathrm{LiHoF}_{4}$ has also been studied by Cooke et al. ${ }^{8}$ which determined the transition temperature $T_{C}=1.53 \mathrm{~K}$. The magnetic interactions are similar to $\mathrm{LiTbF}_{4}$, the dipolar interactions being predominant.

The magnetic susceptibility in $\mathrm{LiErF}_{4}$ was studied by Beauvillain et al. ${ }^{9}$ at ${ }^{3} \mathrm{He}$ temperatures: $\mathrm{LiErF}_{4}$ is antiferromagnetic, $T_{N}=0.38 \mathrm{~K}$ and the magnetic moments are perpendicular to the $\overrightarrow{\mathrm{c}}$ axis in agreement with the theoretical prediction of Misra and Felsteiner. ${ }^{10}$ Beauvillain et al. ${ }^{11}$ have recently studied $\mathrm{LiDyF}_{4}$, which shows properties similar to $\mathrm{LiErF}_{4}$, with $T_{N}=0.581 \pm 0.05 \mathrm{~K}$.

The optical absorption spectra of $\mathrm{LiTbF}_{4}, \mathrm{LiHoF}_{4}$, and $\mathrm{LiErF}_{4}$ have been studied by Christensen ${ }^{12,13}$ and the crystal-field parameters have been determined.

The singular properties of $\mathrm{LiTbF}_{4}$ and $\mathrm{LiHoF}_{4}$ which are good models of dipolar uniaxial ferromagnets are very important for the study of critical phenomena. For Ising dipolar ferromagnets at $d=3$, the critical behavior can be calculated exactly ${ }^{14,15}$ and apart from logarithmic corrections, the classical Landau theory of second-order phase transitions can be applied.

The critical properties of $\mathrm{LiTbF}_{4}$ have been studied by specific-heat, ${ }^{16,17}$ neutron scattering, ${ }^{18-20}$ and opti$\mathrm{cal}^{21}$ measurements. For $\mathrm{LiHoF}_{4}$ the critical properties have been analyzed by susceptibility measurements. ${ }^{22,23}$

We report here, EPR experiments at millimeter and submillimeter frequencies $70<v<600 \mathrm{GHz}$ and 
in magnetic fields up to $60 \mathrm{kG}$ in $\mathrm{LiTbF}_{4} ; \mathrm{LiHoF}_{4}$, and $\mathrm{LiErF}_{4}$. The resonances between the lowest levels have been observed for the three compounds. The temperature dependence of the linewidths has been studied for $\mathrm{LiTbF}_{4}$ and $\mathrm{LiHoF}_{4}$. Resonances due to rare earths and other (iron) residual impurities are also observed but are not analyzed here. EPR experiments on these crystals give information on the crystal-field parameters by the observation of the position in field and by the linewidth dependences of the resonance lines with respect to frequency and temperature. They also give information on the magnetic parameters by the direct observation of the internal fields in the crystal which produce shifts in the resonance positions. These results give the parameters which are necessary for a detailed description of the magnetism in $\mathrm{LiTbF}_{4}, \mathrm{LiHoF}_{4}$, and $\mathrm{LiErF}_{4}$ and for the critical properties of these compounds. We reported earlier some preliminary results. ${ }^{24}$

The $\mathrm{LiTbF}_{4}, \mathrm{LiHoF}_{4}$, and $\mathrm{LiErF}_{4}$ samples used in this study were single crystals grown by Hansen and Laursen by a hydrofluorination method described in Ref. 3. Either spheres or parallelepipeds were used.

The microwave and cryogenic setups have been described elsewhere. ${ }^{4}$

\section{EXPERIMENTAL RESULTS AND INTERPRETATION}

\section{A. $\mathrm{LiTbF}_{4}$}

Two single crystals were used, one parallelipedic (sample 1) the other spherical (sample 2). Only one resonance signal due to the $\mathrm{Tb}^{3+}$ ions was observed in the whole range of frequencies and temperatures. The resonance line was broad at low frequencies $(70<v<135 \mathrm{GHz})$ and narrowed as the frequencies increased. Figure 1 shows recorder traces taken at different frequencies at $T=4.2 \mathrm{~K}$ for sample 1 . The hyperfine structure from the $100 \%$ abundant isotope ${ }^{159} \mathrm{~Tb}$ with nuclear spin $I=\frac{3}{2}$ consists of four equally spaced resonances lines. It was only resolved for sample 2, and only at the highest frequencies (Fig. 2). The spacing between the hyperfince resonance lines is $A=250 \pm 5$ Gauss for $\overrightarrow{\mathrm{H}} \| \overrightarrow{\mathrm{c}}$ in agreement with the values obtained for the dilute material by some of the present authors, ${ }^{25} A=252 \pm 5$ Gauss, and by Laursen and Holmes, $A=252.9 \pm 0.5$ Gauss. $^{3}$

The angular dependence of the resonance line in the $(c, a)$ plane, shown on Fig. 3. displays a very large anisotropy and confirms the Ising behavior. Figure 4 shows the frequency dependence at $4.2 \mathrm{~K}$ of the resonance line as a function of the magnetic field. These frequency field dependences of the resonance signal for $\overrightarrow{\mathrm{H}} \| \overrightarrow{\mathrm{c}}$ have been also studied at $T \leq 1.5 \mathrm{~K}$ in the ferromagnetic phase. At high frequencies

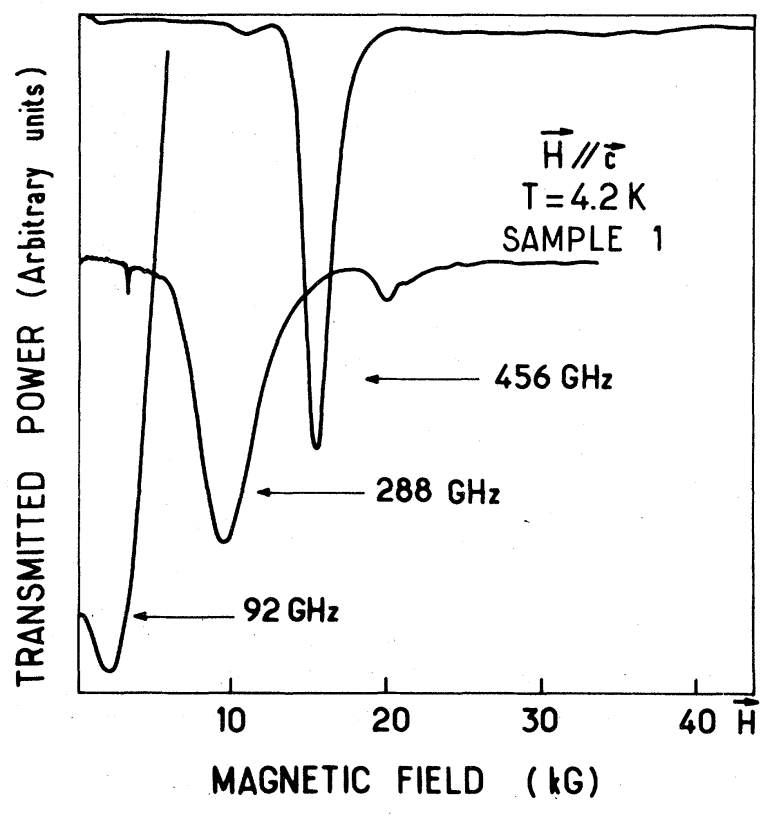

FIG. 1. Recorder traces at three frequencies for sample 1 of $\mathrm{LiTbF}_{4}$. The small resonances observed are due to impurities.

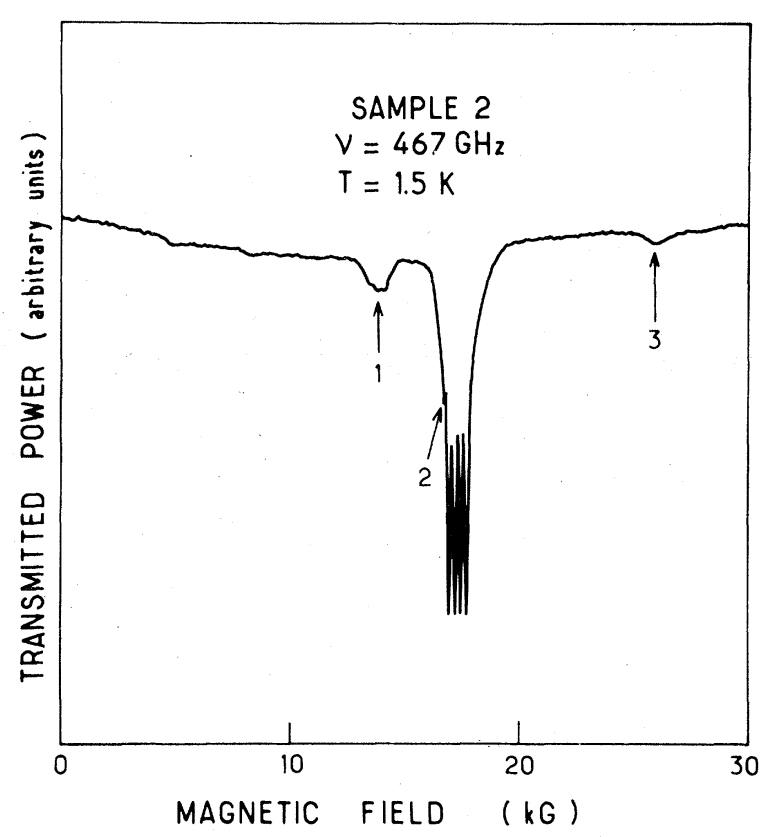

FIG. 2. Recorder traces at $467 \mathrm{GHz}$ for sample 2 of $\mathrm{LiTbF}_{4}$. Resonance 1 and 3 are produced by impurities. Resonance 2 is a forbidden hyperfine transition. 


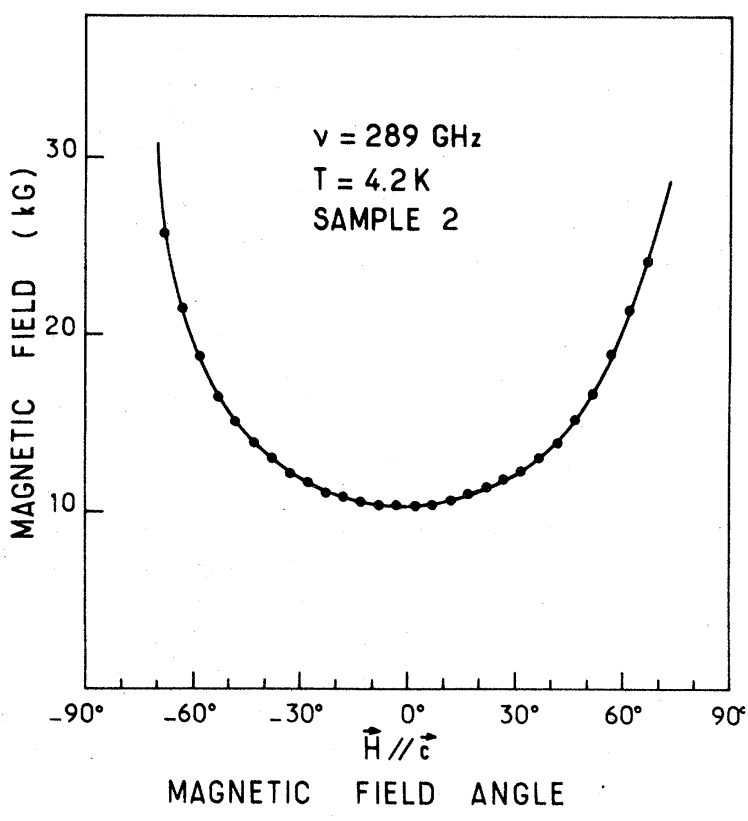

FIG. 3. Angular dependence of the main resonance at $289 \mathrm{GHz}$ for $\overrightarrow{\mathrm{H}}$ in a $(c, a)$ plane in $\mathrm{LiTbF}_{4}$ (sample 2). Dots, experiment; continuous line, a guide to the eye.

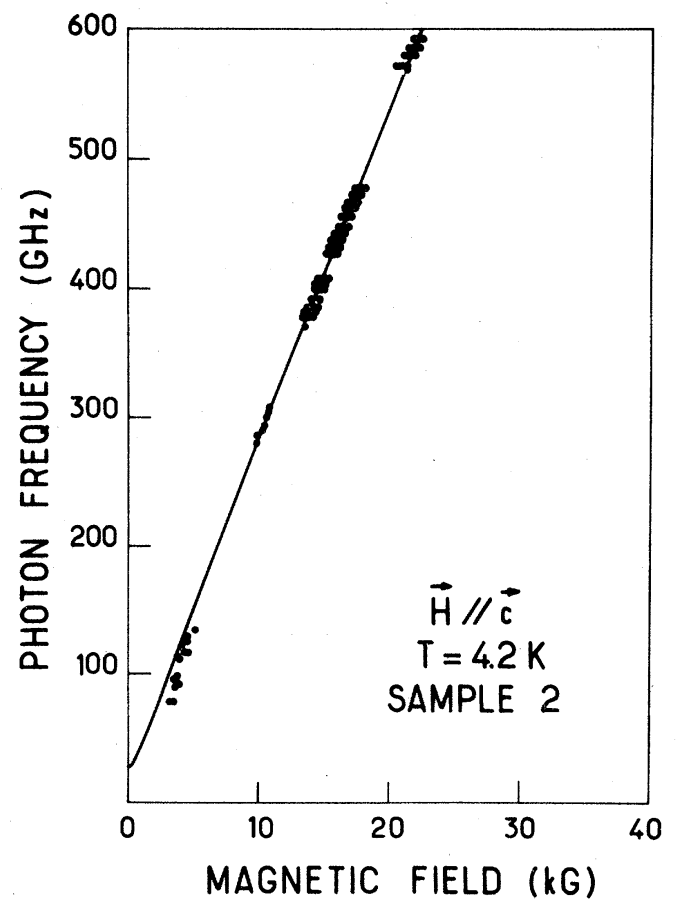

FIG. 4. Frequency vs magnetic field of the resonance for $\overrightarrow{\mathrm{H}} \| \overrightarrow{\mathrm{c}}$ in $\mathrm{LiTbF}_{4}$ (sample 2). Dots, experiment; continuous line, theory. $(\nu>135 \mathrm{GHz})$ for which the sample is saturated, the experimental results are similar to those obtained at $T=4.2 \mathrm{~K}$. At low frequencies $(70<\nu<135 \mathrm{GHz})$, attempts to observe more clearly the ferromagnetic resonance were unsuccessful. This is probably due to the existence of domains below $T_{C} .^{8}$

Figure 5 shows the temperature dependence of the linewidth of the resonance line for two different frequencies in the temperature range $1.4<T<25 \mathrm{~K}$ for sample 2 .

In a crystal field of $S_{4}$ symmetry, the $(2 J+1)$ fold degenerate ground term ${ }^{7} F_{6}$ of the non-Kramers $\mathrm{Tb}^{3+}$ ion $\left(4 f^{8}\right)$ splits into 10 levels ( 3 doublets and 7 singlets),

$$
{ }^{7} F_{6} \rightarrow 3 \Gamma_{1}+4 \Gamma_{2}+3 \Gamma_{3,4} .
$$

The $\Gamma_{3,4}$ doublets correspond to pairs of singlet representations. The optical measurements of Ref. 12 shows that the two lowest levels for two $\Gamma_{2}$ singlets approximately $2 \mathrm{~cm}^{-1}$ apart. The other levels are at $100 \mathrm{~cm}^{-1}$ above.

The corresponding wave functions of these two levels have the general form

$$
\begin{aligned}
\left|\Gamma_{2}^{i}\right\rangle= & \alpha_{i}\left(e^{j \phi_{i}}|+6\rangle-e^{-j \phi_{i}}|-6\rangle\right) \\
& +\beta_{i}\left(e^{j x_{i}}|+2\rangle-e^{-j x_{i}}|-2\rangle\right) .
\end{aligned}
$$

The large experimental value $g_{Z}=17.85$ (see below) suggests that these wave functions are made up mainly of $\left|J_{Z}\right\rangle= \pm 6$ states.

The magnetic field dependence for $\overrightarrow{\mathrm{H}} \| \overrightarrow{\mathrm{c}}$ of these levels can be obtained from the effective spin Hamil-

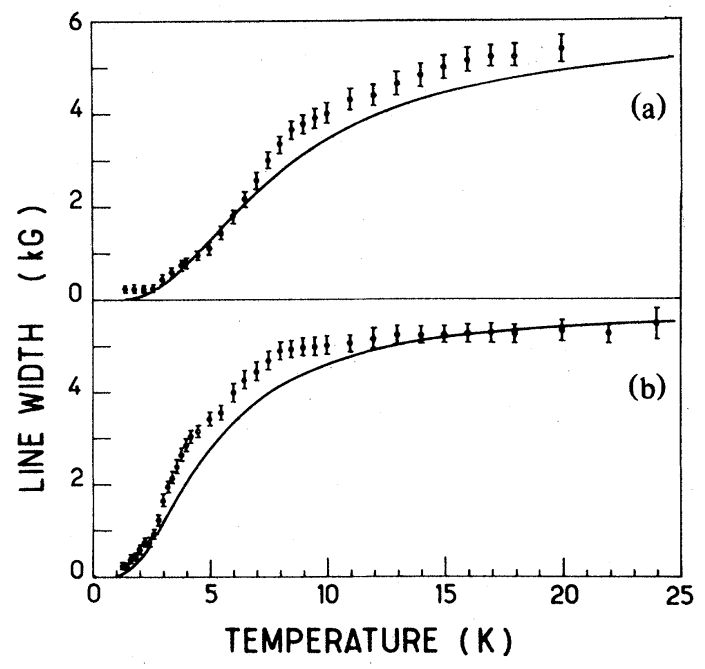

FIG. 5. Linewidth vs temperature of the resonance for $\overrightarrow{\mathrm{H}} \| \overrightarrow{\mathrm{c}}$ in $\mathrm{LiTbF}_{4}$ (sample 2). (a) $\nu=456.5 \mathrm{GHz}$. (b) $\nu=285$ $\mathrm{GHz}$. Dots, experiment; continuous line, theory. 
tonian with $S=\frac{1}{2}, 3$

$$
\mathfrak{H C}=g_{Z} \mu_{\mathrm{B}} H_{Z} S_{Z}+\Delta S_{x}+A S_{Z} I_{Z},
$$

where $\Delta$ is the zero-field splitting of the two $\Gamma_{2}$ levels and $A$ is the hyperfine constant.

The energy levels are given by

$$
E\left(m_{s}, m_{l}\right)=m_{s}\left[\left(g_{Z} \mu_{B} H_{Z}+A m_{l}\right)^{2}+\Delta^{2}\right]^{1 / 2},
$$

where $m_{s}= \pm \frac{1}{2}$ and $m_{I}= \pm \frac{3}{2}, \pm \frac{1}{2}$.

The transitions induced by a microwave field $\Delta m_{s}=1, \Delta m_{l}=0$ are given by

$$
h \nu=\left[\left(g_{Z} \mu_{\mathrm{B}} H_{Z}+A m_{l}\right)^{2}+\Delta^{2}\right]^{1 / 2},
$$

where $H_{Z}$ is the local magnetic field related to the external magnetic field, $H_{0 z}$ by the relation

$$
H_{Z}=H_{0 z}+\left(\lambda-4 \pi N_{c} / V\right) M_{Z},
$$

where $\lambda$ is the molecular-field constant, $N_{c}$ is the demagnetizing factor, $V$ is the gram ionic volume, and $M$ is the magnetization which depends on the temperature, and implicitly depends on the local field via the expression

$$
\begin{aligned}
\frac{M_{Z}}{M_{0}}= & \frac{g_{Z} \mu_{\mathrm{B}} H_{Z}}{\left[\left(g_{Z} \mu_{\mathrm{B}} H_{Z}\right)^{2}+\Delta^{2}\right]^{1 / 2}} \\
& \times \tanh \left(\frac{1}{2 k T}\left[\left(g_{Z} \mu_{\mathrm{B}} H_{Z}\right)^{2}+\Delta^{2}\right]^{1 / 2}\right) .
\end{aligned}
$$

$M_{0}$ is the saturation magnetization. The hyperfine structure is neglected for simplicity. In expression (5), the $\lambda$ constant contains only the dipolar contribution when the spins contribute to the same hyper- fine signal, and the dipolar and exchange contributions when the spins contribute to different hyperfine signals. $^{26-28}$ As the material is primarily dipolar coupled, the $\lambda$ constant has practically the same value as the molecular-field constant obtained from susceptibility experiments. The Curie temperature $\Theta_{C}$ can be determined from the value of the molecular-field constant $\lambda$ according to

$$
\Theta_{C}=\frac{g_{Z} \mu_{\mathrm{B}}}{2 k} \lambda M_{0}
$$

The best-fit values for the parameters of the spin Hamiltonian (2) and for the Curie temperature are given for the two samples used in Table I where they are compared with other experimental results. The low-temperature ferromagnetism in $\mathrm{LiTbF}_{4}$ is described using a fundamental doublet with $g_{Z}=17.85 \pm 0.1$ and $g_{x}=g_{y}=0$. The Ising behavior is a consequence of this anisotropy of the $g$ factor. The dipolar contribution to the Curie constant is predominant. $^{5,6,24}$ The splitting $\Delta$ measured directly in our experiment is the parameter which determines the validity of application of the Ising model. We find $\Delta=30 \pm 10 \mathrm{GHz}$ which is close to the value found in $\mathrm{LiYF}_{4}: \mathrm{Tb}^{3+25}$ The experimental error is large. It is a consequence of the poor precision in the line position due to the large linewidth in the low-frequency region $(70<\nu<135 \mathrm{GHz})$. The ratio $\Delta / k T_{C}=0.50$, $T_{C}=2.86 \mathrm{~K}$, is an important parameter for a detailed description of the system, the critical parameters being possibly affected by a finite $\Delta / k T_{C}$ ratio. ${ }^{19}$

The frequency and temperature dependences of the linewidth have been analyzed in terms of the line

TABLE I. Values for the best-fit parameters obtained in $\mathrm{LiTbF}_{4}$ from the frequency dependence of the resonance line. The values of several of these parameters obtained from other works (see Refs. 3, 5, and 6) are included for comparison.

\begin{tabular}{cccccc}
\hline \hline & $g_{Z}$ & $\left(\lambda-\frac{4 \pi}{V} N_{c}\right) M_{0}(\mathrm{kG})$ & $\Delta(\mathrm{GHz})$ & $\frac{4 \pi}{3} N_{c}$ & $\Theta_{C}(\mathrm{~K})$ \\
\hline Sample 1 & $17.85 \pm 0.1$ & $2.5 \pm 0.1$ & 30 & $4 \pi \times 0.24$ & $3.55 \pm 0.1$ \\
$\begin{array}{c}\text { Sample 2 } \\
\text { LiYF } 4: \mathrm{Tb}^{3+}\end{array}$ & $17.85 \pm 0.1$ & $1.3 \pm 0.1$ & 30 & $4 \pi / 3$ & $3.58 \pm 0.1$ \\
$\begin{array}{c}\text { LiYF } \\
\text { Ref. } 3\end{array}$ & $17.85 \pm 0.1$ & $\ldots$ & $28 \pm 2$ & $\ldots$ & $\ldots$ \\
$\begin{array}{c}\text { Susceptibility } \\
\text { Ref. 5 }\end{array}$ & 17.8 & $\ldots$ & $27.98 \pm 0.06$ & $\ldots$ & $\ldots$ \\
$\begin{array}{c}\text { Neutrons } \\
\text { Ref. 6 }\end{array}$ & $\ldots$ & $\ldots$ & $\ldots$ & $\ldots$ & $3.6 \pm 0.1$ \\
\hline \hline
\end{tabular}


moments theory developed by Kambe and Usui ${ }^{26}$ and McMillan and Opechowski. ${ }^{27}$ Precise experiments of these dependences have been performed by Svare and $\mathrm{Seidel}^{28}$ on $\mathrm{Nd}\left(\mathrm{C}_{2} \mathrm{H}_{5} \mathrm{SO}_{4}\right)_{3} \cdot 9 \mathrm{H}_{2} \mathrm{O}, \mathrm{NiSiF}_{6} \cdot 2 \mathrm{H}_{2} \mathrm{O}$, and $\mathrm{K}_{2} \mathrm{Cu}\left(\mathrm{SO}_{4}\right)_{2} \cdot 6 \mathrm{H}_{2} \mathrm{O}$.

According to these theories, the second moment of the resonance line is

$$
h^{2}\left\langle v^{2}\right\rangle=\frac{\operatorname{Tr}\left\{b(\tilde{\mathfrak{C}})\left[\left(M_{-}, \tilde{\mathcal{C}}\right),\left(\tilde{\mathcal{K}}, M_{+}\right)\right]\right\}}{\operatorname{Tr}\left[b(\tilde{\mathcal{H}})\left(M_{-}, M_{+}\right)\right]},
$$

where $\tilde{\mathcal{H}}$ is the truncated Hamiltonian ${ }^{26}$ and the operators $M_{+}$and $M_{-}$are defined by

$$
\begin{aligned}
& \left\langle n\left|M_{+}\right| n^{\prime}\right\rangle=\left\{\begin{array}{l}
\left\langle n|M| n^{\prime}\right\rangle, \quad E_{n}^{\prime}<E_{n} \\
0, \text { otherwise },
\end{array}\right. \\
& \left\langle n\left|M_{-}\right| n^{\prime}\right\rangle=\left\{\begin{array}{l}
\left\langle n|M| n^{\prime}\right\rangle, \quad E_{n}^{\prime}<E_{n} \\
0, \text { otherwise . }
\end{array}\right.
\end{aligned}
$$

$E_{n}$ are the energies of the unperturbed spin system, $M$ the truncated-microwave-field operator ${ }^{26}$ and $b(x)$ the Boltzmann factors $e^{-x / k T}$.

For $\mathrm{LiTbF}_{4}$, we suppose for simplicity $\Delta=0$. The second central moment is then given through the expression $^{28}$

$$
\begin{aligned}
\left(h^{2}\left\langle\Delta \nu^{2}\right\rangle\right)_{c}= & (16 N)^{-1}\left(2 g_{Z}^{2}+g_{\perp}^{2}\right) \mu_{\mathrm{B}}^{4} \\
& \times\left[1-\tanh ^{2}\left(g_{Z} \mu_{\mathrm{B}} H_{Z} / 2 k T\right)\right] \\
& \times \sum_{i, j}\left(\frac{1-3 \cos ^{2} \theta_{i j}}{r_{i j}^{3}}\right)^{2} .
\end{aligned}
$$

We have fitted the temperature dependence of the linewidths in Fig. 5 by the expression

$$
\Delta H=A\left[1-\tanh ^{2}\left(g_{Z} \mu_{\mathrm{B}} H_{Z} / 2 k T\right)\right]^{1 / 2},
$$

with $A=5.75 \mathrm{kG}$ in good agreement with the value calculated from Eq. (9), $A=6.5 \mathrm{kG}$. The calculated curves in Fig. 5 describe qualitatively the observed temperature dependence.

\section{B. $\mathrm{LiHoF}_{4}$}

Three single crystals of $\mathrm{LiHoF}_{4}$ have been studied. The first two crystals were parallelepipeds (samples 1 and 2) and the third was a sphere.

At a given frequency we observe, in general, several strong resonances. Some of these resonances have a well resolved hyperfine structure consisting of eight resonance lines due to the $100 \%$ abundant isotope ${ }^{165} \mathrm{Ho}$, with nuclear spin $I=\frac{7}{2}$. The spacing between the hyperfine resonance lines is $A=474 \pm 10$ Gauss for $\overrightarrow{\mathrm{H}} \| \overrightarrow{\mathrm{c}}$ in agreement with the value observed in the dilute material $A=479 \pm 7$ Gauss. $^{4}$

The angular dependences of the resonance lines were studied in the $(c, a)$ plane and were similar to those observed in the dilute material. ${ }^{25}$

Figures 6 and 7 show the frequency dependence of the observed resonances as a function of the magnetic field respectively along and perpendicular to the $\overrightarrow{\mathrm{c}}$ axis. On Fig. 6, note that one resonance extrapolates linearly to zero frequency at zero magnetic field.

This is characteristic of transitions between the levels of a doublet. Several other lines extrapolate at 220 $\mathrm{GHz}$ and consequently correspond to transitions between this ground-state doublet and another level at $220 \mathrm{GHz}$ above it. One line extrapolates at about $694 \mathrm{GHz}$ and corresponds to a third level at 694 $\mathrm{GHz}$. Another resonance which extrapolates for zero magnetic field at $220 \mathrm{GHz}$ is observed, the frequency of this resonance is almost exactly at a given field the sum of the frequencies of the resonance lines noted $1 \hookrightarrow 2$ and $1 \leftrightarrow 3$ on Fig. 6 for the same value of the field.

Figure 8 shows the temperature dependence of the linewidth of $1 \rightarrow 2$ for sample 3 in the temperature range $1.4<T<15 \mathrm{~K}$.

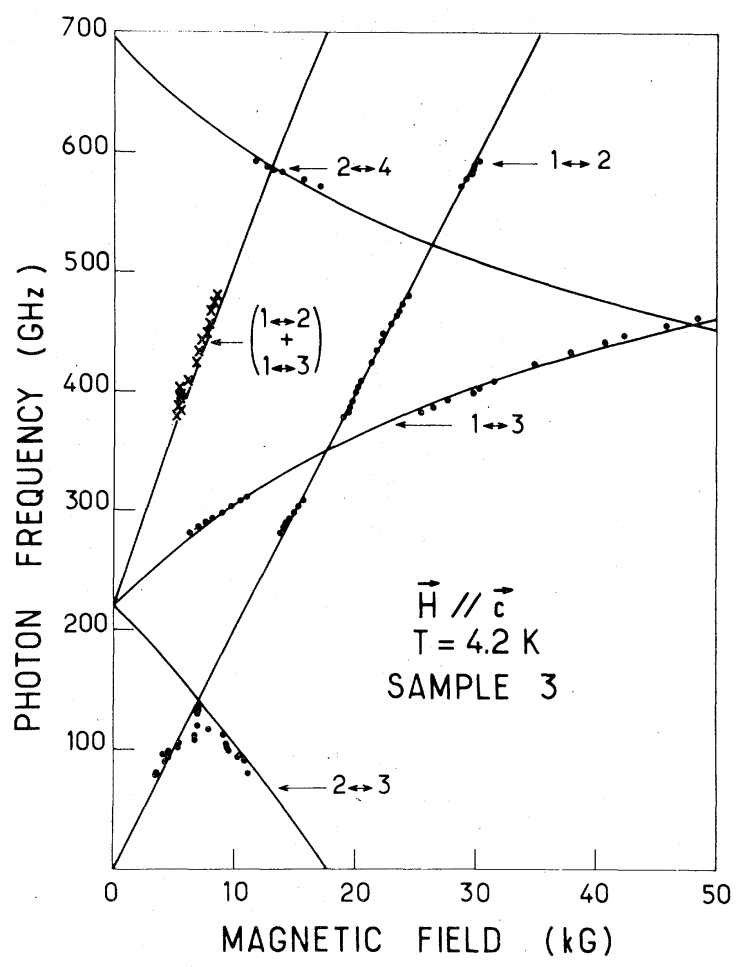

FIG. 6. Frequency vs magnetic field of the resonances for $\overrightarrow{\mathrm{H}} \| \overrightarrow{\mathrm{c}}$ in $\mathrm{LiHoF}_{4}$ (sample 3). Resonance $1 \rightarrow 2$ indicates the transition between the two levels of the fundamental doublet. Resonances $1 \leftrightarrow 3,2 \leftrightarrow 3$ indicate transitions between these two levels and the first excited state and resonance $2 \hookrightarrow 4$ indicates a transition between a level of the fundamental doublet and a second excited state. Dots, experiment; continuous line, theory. 


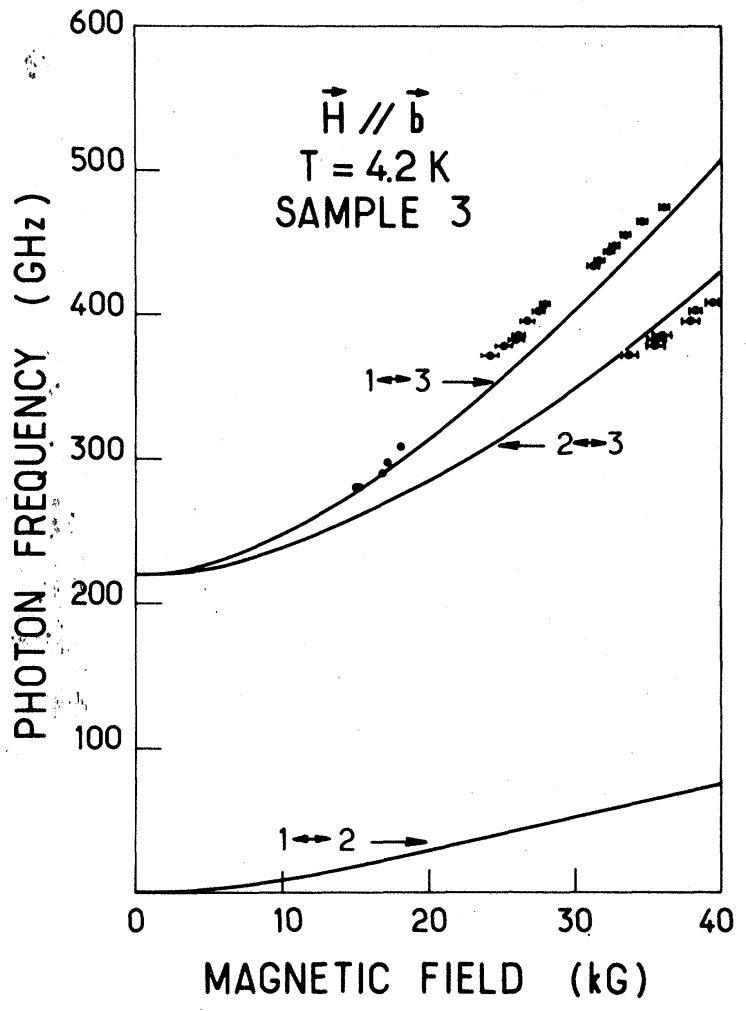

-FIG. 7. Frequency vs magnetic field of the resonances for $\overrightarrow{\mathrm{H}}: \| \overrightarrow{\mathrm{b}}$ in $\mathrm{LiHoF}_{4}$ (sample 3). The resonances are indicated with the same notation as in Fig. 6. Dots, experiment; continuous line, theory.

For other transitions, a careful study of the linewidth dependence cannot be made because of the larger linewidths.

These results can be explained as follows. The $2 J+1$ manyfold ${ }^{5} I_{8}$ of the non-Kramers $\mathrm{Ho}^{3+}$ ion $\left(4 f^{10}\right)$ is split into 13 levels (nine singlets and four

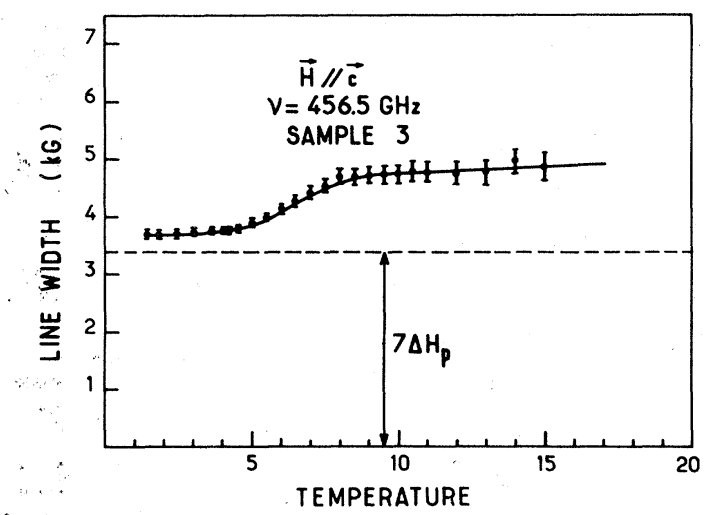

FIG. 8. Linewidth vs temperature of the $1 \rightarrow 2$ resonance for $\overrightarrow{\mathrm{H}} \| \overrightarrow{\mathrm{c}}$ at $\nu=456.5 \mathrm{GHz}$ in $\mathrm{LiHoF}_{4}$ (sample 3). The value $7 \Delta H_{p}$ measures the spacing in field between the first and the eighth hyperfine signal. Dots, experiment; continuous line, a guide to the eye. doublets).

$$
{ }^{5} I_{8} \rightarrow 5 \Gamma_{1}+4 \Gamma_{2}+4 \Gamma_{3,4}
$$

Optical $^{8,13}$ and susceptibility ${ }^{7,8}$ measurements show that the lowest level is a $\Gamma_{3,4}$ doublet and the first two excited levels are two $\Gamma_{2}$ singlets.

The magnetic field dependence of these levels can be obtained from the Zeeman Hamiltonian

$$
H_{Z}=g_{J} \mu_{\mathrm{B}} \overrightarrow{\mathrm{H}} \cdot \overrightarrow{\mathrm{J}}
$$

and from the knowledge of the wave functions of the levels. The Lande factor $g_{J}$ for ${ }^{5} I_{8}$ is equal to $\frac{5}{4}$. More precisely, the energy levels in presence of a magnetic field are calculated from the diagonalization. of the secular equation

$$
\operatorname{det}\left(E_{k l}+\left\langle\Gamma^{k}\left|H_{2}\right| \Gamma^{\prime}\right\rangle-\lambda \delta_{k l}\right)=0
$$

where $k=1, \ldots, 4,\left|\Gamma^{k}\right\rangle$ indicates the $k$ level, $E_{k l}=0$ if $k \neq l$ and $E_{k k}$ is the energy of the $k$ level at $H=0$. We consider only, the four lowest levels. The general structure of the wave functions of these levels is

$$
\begin{aligned}
\left|\Gamma_{3,4}\right\rangle= & \alpha_{1} e^{ \pm \beta_{1} j}| \pm 7\rangle+\alpha_{2} e^{ \pm \beta_{2} J}| \pm 3\rangle \\
& +\alpha_{3} e^{ \pm \beta_{3} j}|\mp 1\rangle+\alpha_{4} e^{ \pm \beta_{4} J}|\mp 5\rangle, \\
\left|\Gamma_{2}^{i}\right\rangle= & \beta_{i}\left(e^{j \phi_{i}}|+6\rangle-e^{-j \phi_{i}}|-6\rangle\right) \\
& +\gamma_{i}\left(e^{j x_{i}}|+2\rangle-e^{-j x_{i}}|-2\rangle\right),
\end{aligned}
$$

where $\alpha_{i}, \beta_{i}$, and $\gamma_{i}$ are real numbers and $i=1,2$.

The study of the transitions between these levels is similar to the case of $\mathrm{Ho}^{3+}$ in the diluted material $\mathrm{LiYF}_{4}: \mathrm{Ho}^{3+}{ }^{4}$ As for $\mathrm{LiTbF}_{3}$, the resonances are shifted by the local magnetic field according to Eq. (5), where the magnetization per ion takes into ac- . count the four lowest levels only. For $\overrightarrow{\mathrm{H}} \| \overrightarrow{\mathrm{c}}$

$$
M_{z}=\frac{\sum_{i} \mu_{i} e^{-E i / k T}}{\sum_{i} e^{-E i / k T}}
$$

and the components of the magnetic moments for these levels are

$$
\begin{aligned}
& \mu\left(\Gamma_{3,4}\right)=\mp \frac{1}{2} g_{Z} \mu_{\mathrm{B}}, \\
& \mu\left(\Gamma_{2}^{i}\right)=\mp \frac{g \mu_{\mathrm{B}}}{2} \frac{g \mu_{\mathrm{B}} H_{Z}}{\left[\left(g \mu_{\mathrm{B}} H_{Z}\right)^{2}+\left(2 \Delta^{\prime}\right)^{2}\right]^{1 / 2}},
\end{aligned}
$$

where $g=2 g_{J}\left\langle\Gamma_{2}^{(2)}\left|J_{Z}\right| \Gamma_{2}^{(1)}\right\rangle$ and where $2 \Delta^{\prime}$ is the splitting at $H=0$ between the two $\Gamma_{2}$ levels.

The magnetization per ion for $\overrightarrow{\mathrm{H}} \| \overrightarrow{\mathrm{b}}$ is more complicated but can again be calculated directly from the partition function as we do below in the $\mathrm{LiErF}_{4}$.

For $\overrightarrow{\mathrm{H}} \| \overrightarrow{\mathrm{c}}$, the analysis of the $\Gamma_{3,4} \leftrightarrow \Gamma_{3,4}$ transition (noted $1 \leftrightarrow 2$ in Fig. 6 ) determines the $g_{Z}$ value and 
TABLE II. Values for the best-fit parameters obtained in $\mathrm{LiHoF}_{4}$ from the frequency dependences of the resonance lines. The values of several of these parameters obtained from other works (Refs. 4, 7, 8, 13, and 23) are included for comparison.

\begin{tabular}{|c|c|c|c|c|c|c|c|c|c|}
\hline & $g_{Z}$ & $\lambda-\frac{4 \pi N_{c}}{V}$ & $M_{0}(\mathrm{kG})$ & $\frac{4 \pi}{3} N_{c}$ & $\Theta_{C}(\mathrm{~K})$ & $E_{1}(\mathrm{GHz})$ & $E_{2}(\mathrm{GHz})$ & $\left\langle\Gamma_{2}^{2}\left|J_{z}\right| \Gamma_{1}^{1}\right\rangle$ & $\left|\left\langle\Gamma_{3,4}\left|J_{x}\right| \Gamma_{2}^{1}\right\rangle\right|$ \\
\hline Sample 1 & $14.1 \pm 0.2$ & $1.5 \pm$ & & $0.23 \times 4 \pi$ & $1.9 \pm 0.1$ & 215 & $\cdots$ & $4.75 \pm 0.2$ & $\cdots$ \\
\hline Sample 2 & $14.1 \pm 0.2$ & $1.9 \pm$ & & $0.21 \times 4 \pi$ & $2.0 \pm 0.1$ & 217 & $\cdots$ & $4.85 \pm 0.1$ & $\cdots$ \\
\hline Sample 3 & $14 \pm 0.2$ & $0.3 \pm$ & & $4 \pi / 3$ & $1.85 \pm 0.1$ & 220 & 694 & $4.85 \pm 0.1$ & 2.3 \\
\hline $\begin{array}{c}\mathrm{LiYF}_{4}: \mathrm{Ho}^{3+} \\
\text { Ref. } 4\end{array}$ & $13.3 \pm 0.1$ & $\cdots$ & & $\cdots$ & $\cdots$ & 204 & 714 & $4.6 \pm 0.1$ & $2.3 \pm 0.1$ \\
\hline $\begin{array}{l}\text { Susceptibility } \\
\text { Ref. } 7\end{array}$ & 13.4 & $\cdots$ & & $\cdots$ & $2.0 \pm 0.2$ & 188 & 815 & 4.71 & 2.43 \\
\hline $\begin{array}{l}\text { Optical suscept. } \\
\text { Ref. } 8\end{array}$ & $\begin{array}{l}13.8 \\
13.6 \pm 0.2\end{array}$ & $\begin{array}{l}\cdots \\
\cdots\end{array}$ & & $\begin{array}{l}\text { several } \\
\text { crystals }\end{array}$ & 1.55 & 222 & 699 & $\cdots$ & $\cdots$ \\
\hline $\begin{array}{l}\text { Optical } \\
\text { Ref. } 13\end{array}$ & 13.79 & $\cdots$ & & $\cdots$ & $\cdots$ & 240 & 780 & 4.74 & 2.31 \\
\hline $\begin{array}{c}\text { Susceptibility } \\
\text { Ref. } 23\end{array}$ & $13.95 \pm 0.15$ & $\cdots$ & & $\cdots$ & 1.65 & 217 & $\cdots$ & $\cdots$ & $\cdots$ \\
\hline
\end{tabular}

the $\lambda$ and $\Theta_{C}$ constants. The analysis of the $\Gamma_{3,4} \leftrightarrow \Gamma_{2}$ transitions (noted $1 \hookleftarrow 3,2 \hookleftarrow 3,1 \leftrightarrow 4$ in Fig. 6) gives the positions in energy of the two $\Gamma_{2}$ singlets $E_{1}, E_{2}$ and also the matrix element $\left\langle\Gamma_{2}^{(2)}\left|J_{Z}\right| \Gamma_{2}^{(1)}\right\rangle$ which governs the field dependence of the $\Gamma_{2}$ singles.

For $\overrightarrow{\mathrm{H}} \| \overrightarrow{\mathrm{b}}$, we determine, from the study of the $\Gamma_{3,4} \leftrightarrow \Gamma_{2}$ transitions, the matrix element $\left|\left\langle\Gamma_{2}^{(1)}\left|J_{x}\right| \Gamma_{3,4}\right\rangle\right|$. The calculated curves for the best-fit parameters are shown in Figs. 6 and 7, respectively.

The values of these parameters are given in Table II and are compared with other results. $4,7,8,13,23$ The low-temperature behavior in $\mathrm{LiHoF}_{4}$ is described by the fundamental doublet with $g_{z}=14.1 \pm 0.2$. If there were no excited states close by, the material would be Ising-like, the same as $\mathrm{LiTbF}_{4}$. The existence of the first excited state at $10.5 \mathrm{~K}$ limits the range of temperatures over which the Ising description can be applied. For $\overrightarrow{\mathrm{H}} \perp \overrightarrow{\mathrm{c}}$ the existence of couplings between this state and the fundamental doublet limits the Ising-like description. The dipolar forces are predominant in the contribution to $\Theta_{C} .4,5,20$ This system can consequently be described as dipolar and Ising-like for low temperatures provided that no magnetic field is applied perpendicular to the $\overrightarrow{\mathbf{c}}$ axis. The critical properties do not seem to be affected by the existence of the first excited state. ${ }^{22,23}$

\section{C. $\mathrm{LiErF}_{4}$}

Three single crystals of $\mathrm{LiErF}_{4}$ were used. The first two crystals were parallelepipedic (samples 1,2) and the third spherical (sample 3 ).

The hyperfine structures due to the different isotopes of $\mathrm{Er}^{3+}$ are not resolved for any temperature or frequency.

The angular dependences of the resonance lines in the $(c, a)$ and $(a, a)$ planes were studied. Figure 9 shows the angular dependence in the $(a, a)$ plane. The resonance lines show an anisotropy in the basal plane with a periodicity of $90^{\circ}$. Similar results had been observed on $\mathrm{LiYF}_{4}: \mathrm{Ho}^{3+}$ on the angular dependences in this plane. ${ }^{25}$

Figures 10-12 show the frequency field dependence of the observed resonances for the magnetic field along the $\overrightarrow{\mathbf{c}}$ axis and along the two directions in the basal plane for which the angular dependence (Fig. 9) shows a maximum and a minimum of the position in field of the resonance line.

In these figures, we observe that several signals extrapolate at zero frequency for zero magnetic fields. These resonances correspond to transitions between doublet components. Several lines extrapolate at 545 $\mathrm{GHz}$ for zero magnetic field and correspond to transi- 


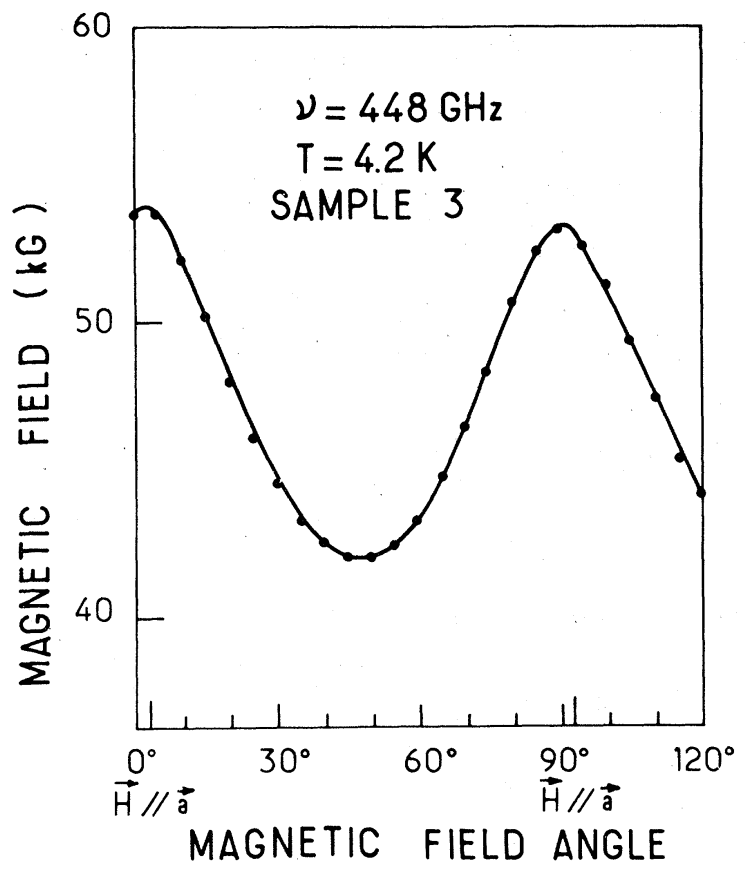

FIG. 9. Angular dependence of the resonance $1 \rightarrow 2$ at $448 \mathrm{GHz}$ for $\overrightarrow{\mathrm{H}} \perp \overrightarrow{\mathrm{c}}$ in $\mathrm{LiErF}_{4}$ (sample 3). Dots, experiment; continuous line, a guide to the eye.

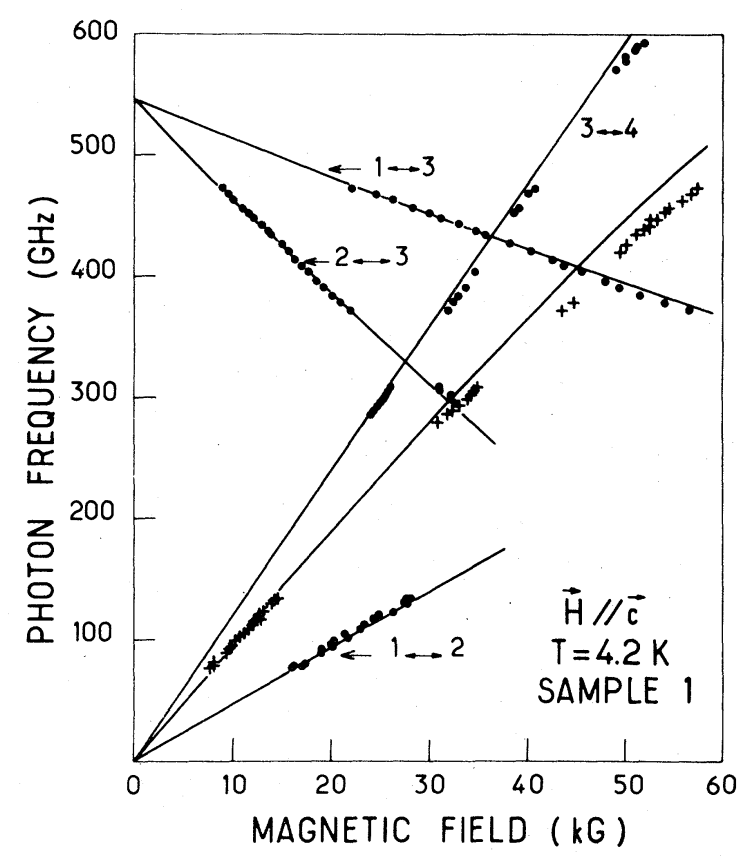

FIG. 10. Frequency vs magnetic field of the resonances for $\overrightarrow{\mathrm{H}} \| \overrightarrow{\mathrm{c}}$ in $\mathrm{LiErF}_{4}$ (sample 1). Resonance $1 \leftrightarrow 2$ indicates the transition between the two levels of the fundamental doublet. Resonances $1 \rightarrow 3$ and $2 \rightarrow 3$ indicate transition between these levels and a level of the first excited doublet. Resonance $3 \multimap 4$ indicates the transition between the two levels of this first excited doublet (see Fig. 13). Dots, experiment; continuous line, theory. tions between two levels $545 \mathrm{GHz}$ apart.

Moreover, two resonances (noted with crosses in Figs. 10-12) were observed at a given field at a frequency which is almost exactly twice the frequency of the resonance lines noted $1 \mapsto 2$. This leads us to the following analysis: the ground term $(2 J+1)$ fold degenerate ${ }^{4} I_{15 / 2}$ of the Kramers ion $\operatorname{Er}^{3+}\left(4 f^{11}\right)$, is split into eight doublets

$$
{ }^{4} I_{15 / 2} \rightarrow 4 \Gamma_{5,6}+4 \Gamma_{7,8}
$$

From the spectroscopic ${ }^{13}$ and the susceptibility measurements ${ }^{7}$ we know the lowest levels. The fundamental level is a $\Gamma_{5,6}$ doublet and the first excited state a $\Gamma_{7,8}$ doublet at $18 \mathrm{~cm}^{-1}$. In our experiment, we observe only the transitions between these two levels. However, the second excited level $\Gamma_{5,6}$ and the third excited level $\Gamma_{7,8}$ must be taken into account for the calculation of the energy levels.

The magnetic field dependence of these levels can be obtained from the Zeeman Hamiltonian (11) with $g_{J}=\frac{6}{5}$ and from the knowledge of the wave functions of the levels. ${ }^{7,13}$

The energy levels are calculated numerically from the diagonalization of the secular equation (12), considering the four lowest doublets. The general struc-

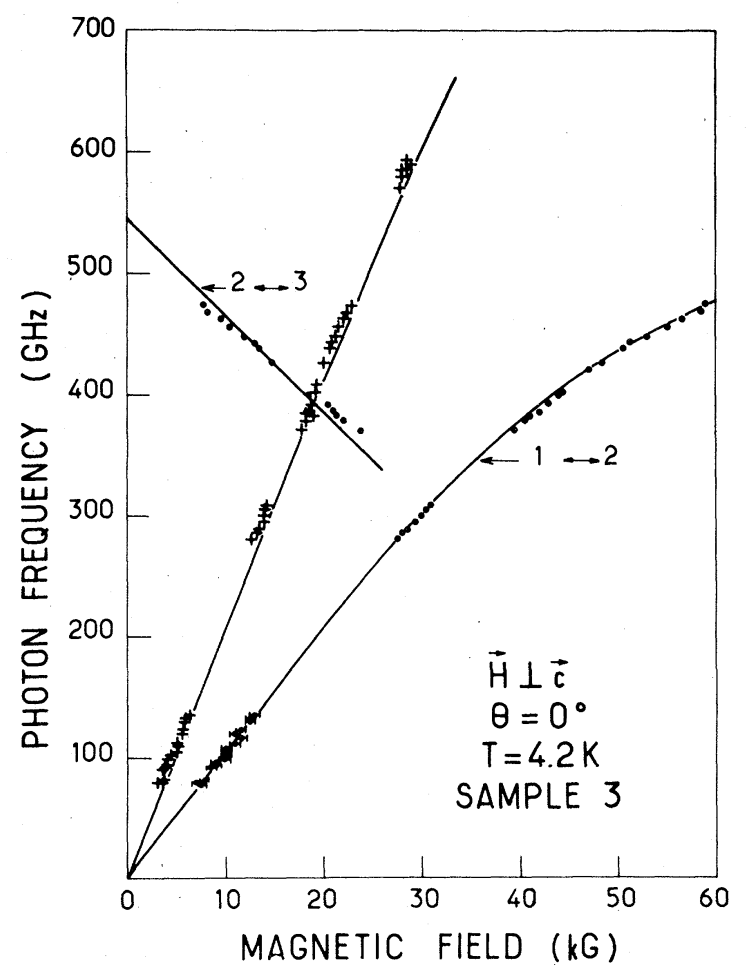

FIG. 11. Frequency vs magnetic field of the resonances for $\overrightarrow{\mathrm{H}} \perp \overrightarrow{\mathrm{c}}$ and $\theta=0^{\circ}$ in $\mathrm{LiErF}_{4}$ (sample 3). The resonances are indicated with the same notation as in Fig. 10. Dots, experiment; continuous line, theory. 


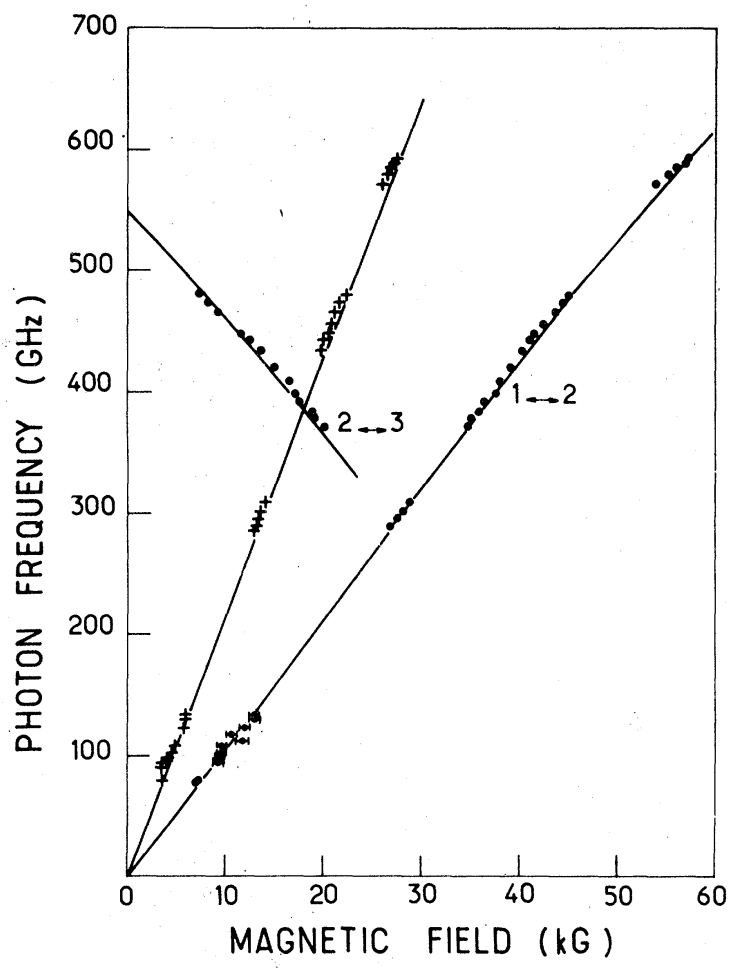

FIG. 12. Frequency vs magnetic field of the resonances for $\overrightarrow{\mathrm{H}} \perp \overrightarrow{\mathrm{C}}$ and $\theta=45^{\circ}$ in $\mathrm{LiErF}_{4}$ (sample 3). The resonances are indicated with the same notation as in Fig. 10. Dots, experiment; continuous line, theory. ture of the wave functions of these levels is

$$
\begin{aligned}
\left|\Gamma_{5,6}\right\rangle= & \alpha_{1} e^{ \pm \beta_{1 j}}\left| \pm \frac{13}{2}\right\rangle+\alpha_{2} e^{ \pm \beta_{2 j}}\left| \pm \frac{5}{2}\right\rangle \\
& +\alpha_{3} e^{ \pm \beta_{3 j}}\left|\mp \frac{3}{2}\right\rangle+\alpha_{4} e^{ \pm \beta_{4 j}}\left| \pm \frac{11}{2}\right\rangle, \\
\left|\Gamma_{7,8}\right\rangle= & \alpha_{1}^{\prime} e^{ \pm \beta_{i j}^{\prime}}\left| \pm \frac{15}{2}\right\rangle+\alpha_{2}^{\prime} e^{ \pm \beta_{2 j}^{\prime}}\left| \pm \frac{7}{2}\right\rangle \\
& +\alpha_{3}^{\prime} e^{ \pm \beta_{3 j}^{\prime}}\left|\mp \frac{1}{2}\right\rangle+\alpha_{4}^{\prime} e^{ \pm \beta_{4 J}^{\prime}}\left|\mp \frac{9}{2}\right\rangle .
\end{aligned}
$$

The resonance lines are shifted in field due to the local magnetic field related to the external magnetic field through the expression.

$$
H=H_{0}+\alpha M_{\text {mass }},
$$

where $M_{\text {mass }}$ is the magnetization per gram. The value of $\alpha$ is taken from susceptibility measurements. $^{9}$

The magnetization per ion is calculated taking into account the four lowest doublets by

$$
M_{\text {ion }}=k T \frac{\partial(\ln Z)}{\partial H},
$$

where $Z=\sum_{n} e^{-E n / k T}$ is the partition function.

The field dependence of the levels is shown in Fig. 13 for $\overrightarrow{\mathrm{H}} \| \overrightarrow{\mathrm{c}}$ and for the two directions in the $\overrightarrow{\mathrm{c}}$ plane corresponding to Figs. 11 and 12.

For $\overrightarrow{\mathrm{H}} \| \overrightarrow{\mathrm{c}}$, we determine from the transition $1 \hookleftarrow 2$, the $g$ value of the fundamental doublet and from transitions $1 \leftrightarrow 3,1 \leftrightarrow 4$, and $3 \leftrightarrow 4$ the $g$ value of the first excited state and the position in zero field of the
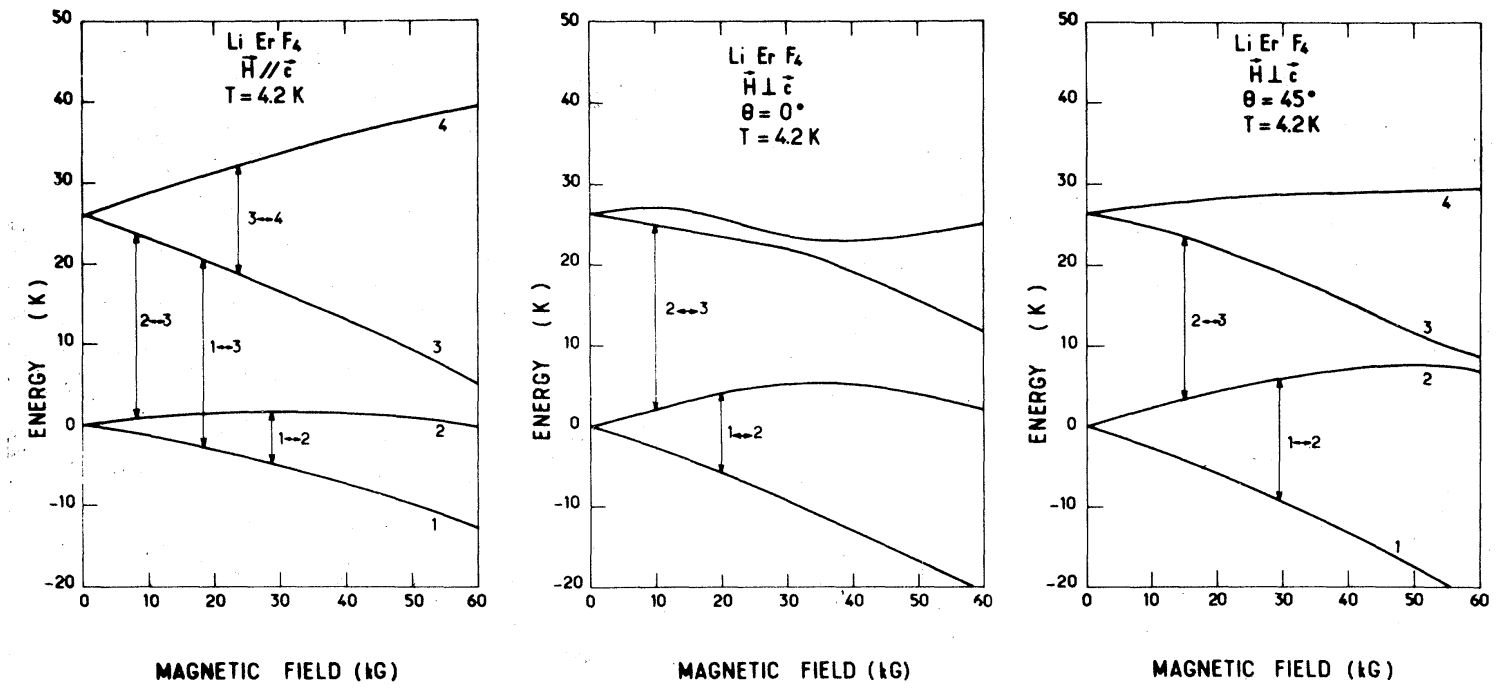

FIG. 13. Scheme of the calculated energy levels in $\operatorname{LiErF}_{4}$. The different transitions are indicated. 
TABLE III. Values for the best-fit parameters obtained in $\mathrm{LiErF}_{4}$ from the frequency dependences of the resonance lines. The values of several of these parameters obtained from other works (Refs. 7, 9, and 13) are included for comparison.

\begin{tabular}{|c|c|c|c|c|c|c|c|}
\hline & $g_{1 c}$ & $g_{2 c}$ & $\alpha$ & $\frac{4 \pi}{3} N_{c}$ & $E_{1}(\mathrm{Ghz})$ & $E_{2}(\mathrm{GHz})$ & $\left\langle\Gamma_{5,6}^{2}\left|J_{2}\right| \Gamma_{5,6}^{1}\right\rangle$ \\
\hline $\begin{array}{l}\text { Samples } 1,2 \\
\overrightarrow{\mathbf{H}} \| \overrightarrow{\mathrm{c}}\end{array}$ & $3.7 \pm 0.2$ & $9.1 \pm 0.2$ & -28.6 & $1.66 \times 4 \pi$ & 546 & 700 & 3.28 \\
\hline $\begin{array}{c}\text { Susceptibility } \\
\text { Ref. } 7\end{array}$ & 3.1 & 8.4 & $\cdots$ & $\cdots$ & 564 & 920 & 3.28 \\
\hline $\begin{array}{l}\text { Susceptibility } \\
\text { Ref. } 9\end{array}$ & 3.5 & $\cdots$ & -40.7 & $4 \pi / 3$ & $\cdots$ & $\ldots$ & $\cdots$ \\
\hline $\begin{array}{l}\text { Optical } \\
\text { Ref. } 13\end{array}$ & 4.2 & 7.9 & $\ldots$ & $\ldots$ & 540 & 600 & 3.09 \\
\hline & $g_{11}$ & $g_{21}$ & $\alpha_{1}$ & $\frac{4 \pi}{3} N_{c}$ & $E_{1}$ & $E_{2}$ & $\left|\left\langle\Gamma_{5,6}^{1}\left|J_{x}\right| \Gamma_{7,8}^{1}\right\rangle\right|$ \\
\hline $\begin{array}{c}\text { Sample } 3 \\
\overrightarrow{\mathrm{H}} \perp \overrightarrow{\mathrm{c}}\end{array}$ & $8.7 \pm 0.2$ & $4.6 \pm 0.2$ & -23.0 & $\cdots$ & 546 & 700 & 2.69 \\
\hline $\begin{array}{l}\text { Susceptibility } \\
\text { Ref. } 7\end{array}$ & 8.2 & 4.6 & $\cdots$ & $\ldots$ & 564 & 920 & 2.24 \\
\hline $\begin{array}{c}\text { Susceptibility } \\
\text { Ref. } 9\end{array}$ & 9.2 & $\ldots$ & -23.0 & $4 \pi / 3$ & $\cdots$ & $\cdots$ & $\cdots$ \\
\hline $\begin{array}{l}\text { Optical } \\
\text { Ref. } 13\end{array}$ & 7.8 & 2.05 & $\cdots$ & $\ldots$ & 540 & 600 & 1.57 \\
\hline
\end{tabular}

two excited levels $E_{1}, E_{2}$. The values of the matrix elements $\left\langle\Gamma_{5,6}^{1}\left|J_{Z}\right| \Gamma_{5,6}^{2}\right\rangle,\left\langle\Gamma_{7,8}^{1}\left|J_{Z}\right| \Gamma_{7,8}^{2}\right\rangle$ and the $g$ values for the two upper doublets are taken from Ref. 7. The best fit of these parameters is given in Table III and compared with other results.

For $\overrightarrow{\mathrm{H}} \perp \overrightarrow{\mathrm{c}}$, the $g$ value of the fundamental doublet and the coupling $\left\langle\Gamma_{5,6}^{1}\left|J_{x}\right| \Gamma_{7,8}^{1}\right\rangle$ are determined from transition $1 \leftrightarrow 2$. The $g$ value of the first excited doublet is determined from transition $2 \leftrightarrow 3$. The $g$ values of the other doublets and other couplings are taken as in Ref. 7. The values of these parameters are given in Table III. The calculated curves with these parameters are shown in Figs. 10-12.

The low-temperature behavior in $\mathrm{LiErF}_{4}$ is described by the fundamental doublet $g_{1 Z}=3.7$ and $g_{1 \perp}=8.7$. These values are rather different from those of Refs. 7 and 12 but are close to those obtained from susceptibility measurements at low temperatures. 9

$\mathrm{LiErF}_{4}$ is a $X Y$ dipolar antiferromagnet $\left(g_{11} / g_{1 z}=2.35\right)$, whereas $\mathrm{LiTbF}_{4}$ and $\mathrm{LiHoF}_{4}$ are Ising dipolar ferromagnets. The dipolar forces and the exchange forces are of the same order. ${ }^{9}$ It seems therefore quite interesting to study the critical param- eters of $\mathrm{LiErF}_{4}$ and compare them with those obtained in $\mathrm{LiTbF}_{4}$ and $\mathrm{LiHoF}_{4}$.

Besides the main resonances, we observe in Figs. 10-12 transitions at twice the energy of that noted $1 \leftrightarrow 2$ in these figures. We attribute these resonance lines to transitions between the levels of two neighbor spins coupled by dipolar forces.

The Hamiltonian for the systems of two spins 1 and 2 is, considering only the fundamental doublet $\left(S_{1}=\frac{1}{2}, S_{2}=\frac{1}{2}\right)$

$H_{12}=\mu_{\mathrm{B}}\left(\overrightarrow{\mathrm{H}} \cdot \vec{g} \cdot \overrightarrow{\mathrm{S}}_{1}\right)+\mu_{\mathrm{B}}\left(\overrightarrow{\mathrm{H}} \cdot \vec{g} \cdot \overrightarrow{\mathrm{S}}_{2}\right)+\overrightarrow{\mathrm{S}}_{1} \cdot \vec{D} \cdot \overrightarrow{\mathrm{S}}_{2}$,

with

$$
D_{12}^{\alpha \beta}=\frac{\mu_{\mathrm{B}}^{2} g^{\alpha \alpha} g^{\beta \beta}}{r_{12}^{3}}\left(\delta_{\alpha \beta}-\frac{3 r_{12}^{\alpha} r_{12}^{\beta}}{r_{12}^{2}}\right),
$$

where $\alpha=x, y, z . \quad g^{\alpha \alpha}$ and $g^{\beta \beta}$ are the $g$ values of the fundamental doublet. $r_{12}$ the distance between the spins 1 and 2.

Taking into account only the secular part of the dipolar interaction ${ }^{29}$ the energy levels can be easily calculated. The levels are characterized by the total spin $\overrightarrow{\mathrm{S}}=\overrightarrow{\mathrm{S}}_{1}+\overrightarrow{\mathrm{S}}_{2}$ and the allowed transitions are those with 
$\Delta S_{Z}= \pm 1$. The effect of the nonsecular part of the dipolar interaction is to mix these states, and it is then possible to observe transitions $\Delta S_{Z}= \pm 2$ at a frequency almost exactly double that of the $\Delta S_{Z}= \pm 1$ transitions. $^{29}$

In $\mathrm{LiTbF}_{4}$, similar resonances cannot be observed because $g_{\perp}=0$, and consequently the nonsecular part of the dipolar interaction is zero.

In $\mathrm{LiHoF}_{4}$, the resonance observed at frequency sum of the $1 \leftrightarrow 2$ and $1 \leftrightarrow 3$ transition, can also be attributed to a transition between the levels of two nearest-neighbor spins and considering, in this case, the three lowest levels of the $\mathrm{Ho}^{3+}$ ion.

\section{SUMMARY}

The $g$ factor and the $\Delta$ splitting of the fundamental doublet of $\mathrm{LiTbF}_{4}$ are determined directly from EPR experiments in a large range of frequencies and magnetic fields. The local field around the $\mathrm{Tb}^{3+}$ ion is determined and the Curie constant calculated. These parameters describe the magnetic behavior of the crystal at low temperatures.

The temperature dependence of the linewidth of the resonance line is observed for $1.4<T<25 \mathrm{~K}$ and analyzed in terms of the theory of line moments.

In $\mathrm{LiHoF}_{4}$, we determine the $g$ factor of the fundamental doublet, the energy of the first two excited states, and the coupling between all these levels in a magnetic field parallel and perpendicular to the tetragonal $\vec{c}$ axis. The local field and the Curie constant are also determined. The temperature dependence of the linewidths was observed.

$\mathrm{LiTbF}_{4}$ and $\mathrm{LiHoF}_{4}$ are quasi-ideal examples of dipolar Ising ferromagnets. Small deviations from the ideal behavior are produced by the single-ion parameters, which are determined in this work.

The $g$ factors, and the couplings between the first two doublets are determined in $\mathrm{LiErF}_{4}$ which is described as an $X Y$ dipolar antiferromagnet.

Some transitions observed in $\mathrm{LiErF}_{4}$, attributed to transitions between the levels of two neighbor ions, are analyzed.

\section{ACKNOWLEDGMENTS}

It is our pleasure to thank Dr. J. P. Renard for many helpful discussions and for his critical reading of the manuscript.
*Also, Université Pierre et Marie Curie (Paris VI).

†Laboratoire Associé au Centre National de la Recherche Scientifique.

${ }^{1}$ E. P. Chiklis, C. S. Naiman, R. C. Folweiler, D. R. Gabbe, H. P. Jenssen, and A. Linz, Appl. Phys. Lett. 19, 119 (1971).

2J. P. Sattler and J. Nemarich, Phys. Rev. B 4, 1 (1971).

${ }^{3}$ I. Laursen and L. M. Holmes, J. Phys. C $\underline{7}, 3765$ (1974).

${ }^{4}$ J. Magariño, J. Tuchendler, J. P. D'Haenens, and A. Linz, Phys. Rev. B $\underline{13}, 2805$ (1976).

${ }^{5}$ L. M. Holmes, T. Johansson, and H. J. Guggenheim, Solid State Commun. 12, 993 (1973)

${ }^{6}$ L. M. Holmes, J. Als-Nielsen, and H. J. Guggenheim, Phys. Rev. B 12, 180 (1975).

${ }^{7}$ P. E. Hansen, T. Johansson, and R. Nevald, Phys. Rev. B 12,5315 (1975).

${ }^{8}$ A. H. Cooke, D. A. Jones, J. F. A. Silva, and M. R. Wells, J. Phys. C 8,4083 (1975); J. E. Battison, A. Kaster, M. J. M. Leask, J. B. Lowry, and B. M. Wanklyn, J. Phys. C $\underline{8}$, 4089 (1975).

${ }^{9}$ P. Beauvillain, J. P. Renard, and P. E. Hansen, J. Phys. C 10, L709 (1977).

${ }^{10}$ S. K. Misra and J. Felsteiner, Phys. Rev. B $\underline{15}, 4309$ (1977).

${ }^{11}$ P. Beauvillain and J. P. Renard (unpublished).

${ }^{12}$ H. P. Christensen, Phys. Rev. B 17, 4060 (1978).

${ }^{13}$ H. P. Christensen (unpublished).

${ }^{14}$ A. I. Larkin and D. E. Khemel'nitskii, Sov. Phys. JETP 29
1123 (1969).

${ }^{15}$ A. Aharony, Phys. Rev. B 8, 3363 (1973).

${ }^{16}$ L. M. Holmes, F. Hulliger, H. J. Guggenheim, and J. P. Maita, Phys. Lett. A 50, 163 (1974).

${ }^{17} \mathrm{G}$. Ahlers, A. Kornblit, and H. J. Guggenheim, Phys. Rev. Lett. 34, 1227 (1975).

18J. Als-Nielsen, L. M. Holmes, and H. J. Guggenheim, Phys. Rev. Lett. 32, 610 (1974).

${ }^{19} \mathrm{~J}$. Als-Nielsen, L. M. Holmes, F. Krebs Larsen, and H. J. Guggenheim, Phys. Rev. B 12, 191 (1975).

20J. Als-Nielsen, Phys. Rev. Lett. 37, 1161 (1976).

${ }^{21}$ J. A. Griffin, J. D. Litster, and A. Linz, Phys. Rev. Lett. 38, 251 (1977).

22P. Beauvillain, J. P. Renard, I. Laursen, and P. J. Walker, J. Phys. (Paris) 39, C6-745 (1978).

${ }^{23}$ P. Beauvillain, J. P. Renard, I. Laursen, and P. J. Walker, Phys. Rev. B 18,3360 (1978).

24J. Magariño, J. Tuchendler, and P. E. Hansen, Physica (Utrecht) B 86-88, 1223 (1977).

25J. Magariño and J. Tuchendler (unpublished).

${ }^{26}$ K. Kambe and T. Usui, Prog. Theor. Phys. (Kyoto) $\underline{8}, 302$ (1952).

${ }^{27}$ M. McMillan and W. Opechowski, Can. J. Phys. $\underline{38}, 1168$ (1960).

28I: Svare and G. Seidel, Phys. Rev. 134, A172 (1960).

${ }^{29} \mathrm{~A}$. Abragam and B. Bleaney, Electron Paramagnetic Resonance of Transition Ions, (Clarendon, Oxford, 1970). 